\title{
SOME Mechanisms for MANAging Aggressive BEHAVIOR IN GROUP ROBOTICS
}

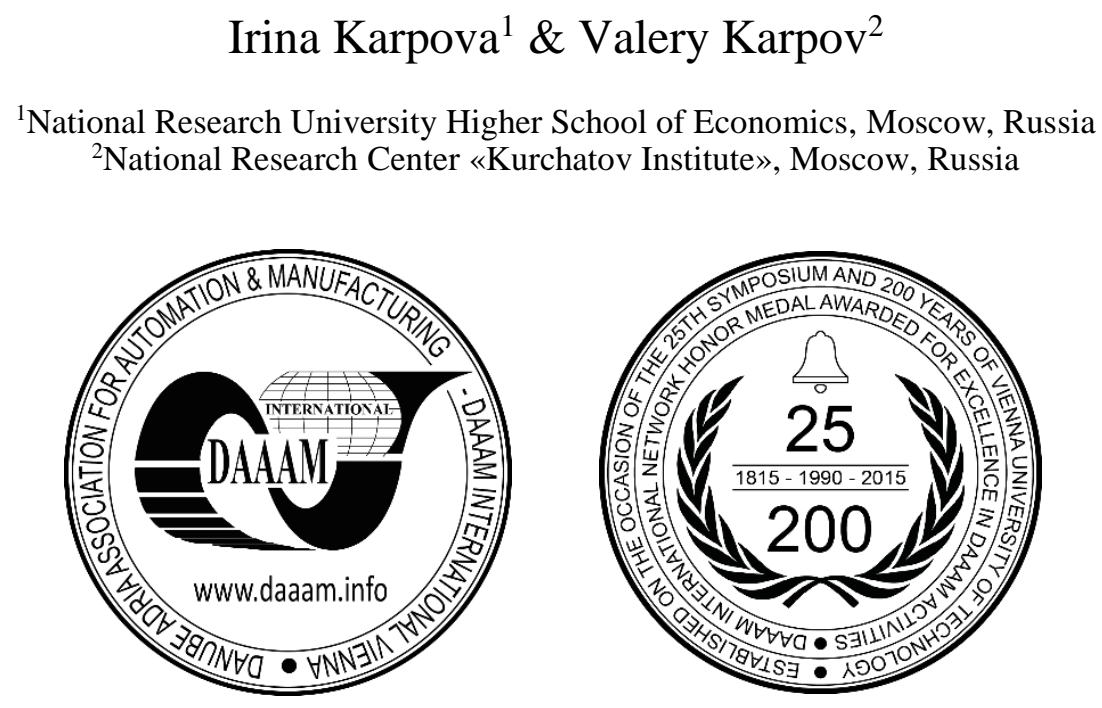

This Publication has to be referred as: Karpova, I[rina] \& Karpov, V[alery] (2018). Some Mechanisms for Managing Aggressive Behavior in Group Robotics, Proceedings of the 29th DAAAM International Symposium, pp.0566-0573, B. Katalinic (Ed.), Published by DAAAM International, ISBN 978-3-902734-20-4, ISSN 1726-9679, Vienna, Austria DOI: $10.2507 / 29$ th.daaam.proceedings.082

\begin{abstract}
Possible methods of implementing aggression as one of the mechanisms in the formation of social behavior in groups of robots are discussed. Aggression is seen as a way to resolve conflicts over resources. The features of the aggressive behavior of eusocial insects (ants) are used as a basis. The aggressive component is integrated into the need-emotional architecture of the robot control system, which is presented as a hybrid neuro-production system. The proposed mechanism can be used as a base for implementing various models of social behavior in group robotics.
\end{abstract}

Keywords: group robotics; social behavior models; aggressive behavior; emotional control system

\section{Introduction}

One of the approaches to the creation of group robotics systems being actively developed is the implementation of social behavior models as unified methodological bases [1]. The main idea of this approach is as follows. The appearance of emergent properties is possible in a robot group if the group is a social community. Therefore, we must study and realize animal social behavior mechanisms, such as cohesion, domination, contagious behavior, etc. [2]. We suppose that these biologically inspired methods will allow for the solving of a wide range of tasks in group robotics.

Ethologists believe that agonistic or aggressive behavior is one of the main mechanisms of social behavior [2]. We need to explore the basic mechanisms of aggressive behavior, because we will use them to realize some social behavior models in group robotics. In the framework of evolutionary theory, aggressive behavior is understood to encourage survival of the fittest, disperse populations, aid adaptation to threatening environments, and generally improve the probability of individual and species survival [3].

Aggressive behavior is an integral part of various social behavior models; therefore, in the present study, the implementation of this mechanism is essential to create higher-level models. There are works in which approaches based on aggressive animal behavior are used to solve certain robotics tasks. In [4], two robots determine which of them will first pass along a narrow corridor and which will give way. 
They evaluate their losses, and the robot gives way if its losses are fewer. This robot is considered less aggressive. The development of this approach for a robot team is presented in [5]. In addition, the term "aggression" is sometimes applied in multi-agent systems. Aggression can be seen as a way of resolving conflicts during the formation of coalitions [6]. In [7], an agent evaluates the usefulness of the resource over which the conflict is taking place. If the usefulness of the resource is greater than the loss as a result of the conflict, the agent gets into a fight.

In [8], another approach was proposed. In ants, actual aggressiveness depends on the distance from its "nest" and the number of other individuals of the species. The author proposes an agent aggressive behavior model depending on these two parameters. This model is designed to solve the problem of dividing the territory between two robot groups. Unfortunately, each of these methods allows solving only one specific task, and they are not suitable for implementing different models of social behavior.

We are interested in a constructive and biologically based aggressive behavior model that can be applied to solve specific problems in group robotics. The constructability denotes that the model can be used in a robot control system. It is worth noting that we mean the interpretation of the agent's actions by an outside observer when we use the term "aggression" or "aggressive behavior." This will allow us to describe naturally the robot's behavioral aspects.

Our purpose is to determine the components of a control system that influences robot behavior so an outside observer considers the behavior to be aggression. The rest of the paper is organized as follows. Section 2 describes the general features of aggressive behavior in animals, particularly ants. Section 3 presents the architecture of a robot control system that uses higher-level emotional mechanism forms. It is argued that the aggressive behavior can be implemented in the control system with a few additional parameters. Section 4 describes the experiments aimed at detecting changes in an agent's behavior depending on the current level of aggressiveness. Section 5 summarizes the material and concludes with a brief outlook on further research.

\section{Aggressive behavior}

Aggressiveness is a special form of agonistic behavior that includes threats, displays, retreats, placation, and conciliation. Aggression is an integral part of many types of social behavior [9], [10], [2], in particular:

- The maintenance of a population's spatial structure

- Mating behavior

- The care of offspring

- The maintenance of a dominance hierarchy among cooperating individuals in a society

- The maintenance of group (family) homeostasis

Aggressive behavior is difficult to define. Nelson writes, "Lack of agreement regarding definitions of aggressive behavior has been a significant impediment to the progress of research in this area" [11]. In our view, the most constructive definition is that aggression is "a consequence of a conflict of interest between an individual, a couple or a group" [12]. Aggression is seen as a means of conflict resolution. This allows us to use the concept of aggression to resolve conflicts between individuals or groups by directly influencing the object causing the conflict.

Generally, aggression contributes to survival of the species [13], usually occurring when an opponent appears. We consider only intraspecific aggression, in which animals attack members of their own species. This type of aggression shapes social behavior.

Agonistic behavior consists of threats, aggression, and submission. Intraspecific aggression rarely proceeds to the fight phase, and it is often limited to demonstrating threats. The threats appear as fixed action patterns (FAPs) [2], which are highly stereotyped models of the behavior characteristic of a particular species. A FAP is a ritual that replaces the fight, and it does no harm to rivals. In the process of evolution, mechanisms that suppress aggressive behavior are developed, either by eliminating the cause (rival, obstacle, etc.) or by another individual demonstrating subordination.

When studying aggression for use in group robotics, we first focus on the behavior of ants as representatives of eusocial insects. Aggression is part of the mechanism that ensures the isolation of the insect community as a whole [14]. The manifestation of aggression in ants can be divided into two classes [15]:

1) intra-family aggression (stimulating effects, formation of subordinate structures, competition between workers of the same family, control of family composition) and 2) extra-family aggression (protection of brood and reproductive individuals, other workers, nest, fodder area).

Each anthill has its own specific odor, and ants distinguish an individual belonging to their anthill from an individual belonging to another anthill based on odor. The first ones we will call as "friends" and the second ones as "aliens" or "foe". Extra-family aggression depends on the distance to the "nest" and on the number of "friends" nearby.

Aggressiveness among ants increases with age. The most aggressive and experienced ants (particularly wood ants of the Formica rufa group) are observers on an anthill dome. In coordinating the actions of ants, imitative behavior plays a main role. For example, ant observers take an aggressive pose or attack when a threat arises. Other ants react to these characteristic movements and poses, and they almost instantly switch to defence mode [15].

Subordination structures are formed when it is necessary to coordinate the ant group's actions. For example, if a recognized leader of the foraging group exists, then the subordinate individuals simply imitate his actions. 
If there is no leader, the most active foragers struggle among themselves for leadership. Subsequently, a leader is recognized by other foragers. It is often impossible to determine unambiguously whether an action is aggressive. The same actions can be considered in one situation as an aggressive manifestation and in another as non-aggressive. Only an observer determines whether an action is aggressive by considering the context in which it occurs. Now, consider how the above can be reflected in the structure of the robot control system.

\section{Generalized model of aggressive behavior}

In the process of functioning, the robot evaluates its own state and the state of the environment, and it carries out certain actions that are determined by its needs and capabilities. Let there be a target state $\left(S_{T}\right)$ and a current $\left(S_{C}\right)$ state. The robot moves from state to state with the action Act:

$$
S_{c} \stackrel{\text { Act }}{\rightarrow} S_{T}
$$

Let us define what we mean by aggressive actions. First, there must be a certain target object $O^{A}$ at which the aggression is directed. Second, we will assume the purpose of the impact is the removal of this object from the scene. This can be written as follows:

$$
S_{T}=S_{c} \backslash O^{A}
$$

We will call the action Act (1) aggressive if it is aimed at eliminating the cause of the inconsistency of the current state with the target state by rendering the destructive impact on the object. In other words, aggressive behavior enables the transformation of the current state to eliminate the cause of the discrepancy.

We view aggressive behavior as a way of resolving conflict, which is understood as the most radical way to resolve contradictions that arise in the process of interaction. Usually, a conflict is accompanied by negative emotions [16]; therefore negative emotions can be a sign of conflict and an internal cause of aggression.

Emotions are one of the main mechanisms for regulating behavior, and they are responsible for contrasting sensory perception and for stabilizing behavior. They are not only characteristic of living organisms, but they are also applicable to technical systems. In [17], architecture for the emotional control system of the agent was proposed. Emotions in this system are the basis for controlling the behavior of the agent, determining the overall assessment of the current situation. This approach is based on the need-information theory of emotions by Simonov [18].

In accordance with Simonov's theory, emotions are an assessment of the value of the current need and the possibility of satisfying it. The brain evaluates this possibility based on genetic predisposition and previously obtained individual life experience. This can be expressed as follows:

$$
E=f\left(N, p\left(I_{\text {need }}, I_{\text {has }}\right)\right)
$$

where $E$ is the emotion (its force, quality, and sign); $N$ is the force and quality of the current need; $\boldsymbol{p}\left(I_{\text {need }}, I_{\text {has }}\right)$ is the estimate of the possibility of satisfying the need using the inherent and gained life experience; $I_{\text {need }}$ is the information about the method to satisfy the need; and $I_{\text {has }}$ is information about the means, resources, and time the subject has presently at its disposal. The difference between the need and possibilities of their satisfaction determines the emotional estimate of the current situation. If we have some needs and the possibilities of satisfying them are sufficient, then we have a positive emotional estimate. Otherwise, the emotions are negative.

The emotional component is enough to realize simple forms of agent behavior. However, in real nature, animal behavior is characterized by great diversity. For example, different individuals will act differently with the same level of needs in the same situation: one will attack, while the other will run away. First, it depends on the aggression level. A more aggressive individual more often chooses an attack than flight or submission. Even the same individual can behave differently in the same situations. The individual's needs have not changed, the strength has not changed, but the individual escapes instead of attacking, depending on the current aggression level.

The condition for the initiation of any action is negative emotions associated with unrealized needs. In the robot control system, the state of each action block is characterized by its private emotion $E_{i}$. The condition for choosing an action is determined by the emotional state of the agent:

$$
C_{a}=C_{a}(E), E=\left\{E_{i}\right\}
$$

An independent observer can interpret some of these actions as aggressive.

As a universal tool for implementing behavioral procedures, finite automata are used [19]. Each automate implements one FAP, and the agent's control system has two levels: the behavior control level and the level of the FAP's implementation. An important issue is the choice of the object of aggression. For example, an object of aggression may be the object with which an action is associated, characterized by minimal (maximum negative) emotion: 


$$
O_{a g g r}=O_{i}: E_{i}=\min E
$$

We view aggression as a regulator of social behavior. Therefore, of all objects that cause negative emotions, only other agents are selected.

Let us consider in more detail the behavior patterns in which the aggressive component is present in an explicit form: territorial behavior (protection of the forage site) in Figure 1 and dominance and self-preservation in Figure 2. Dashed lines indicate negative connections: they play the role of a brake.

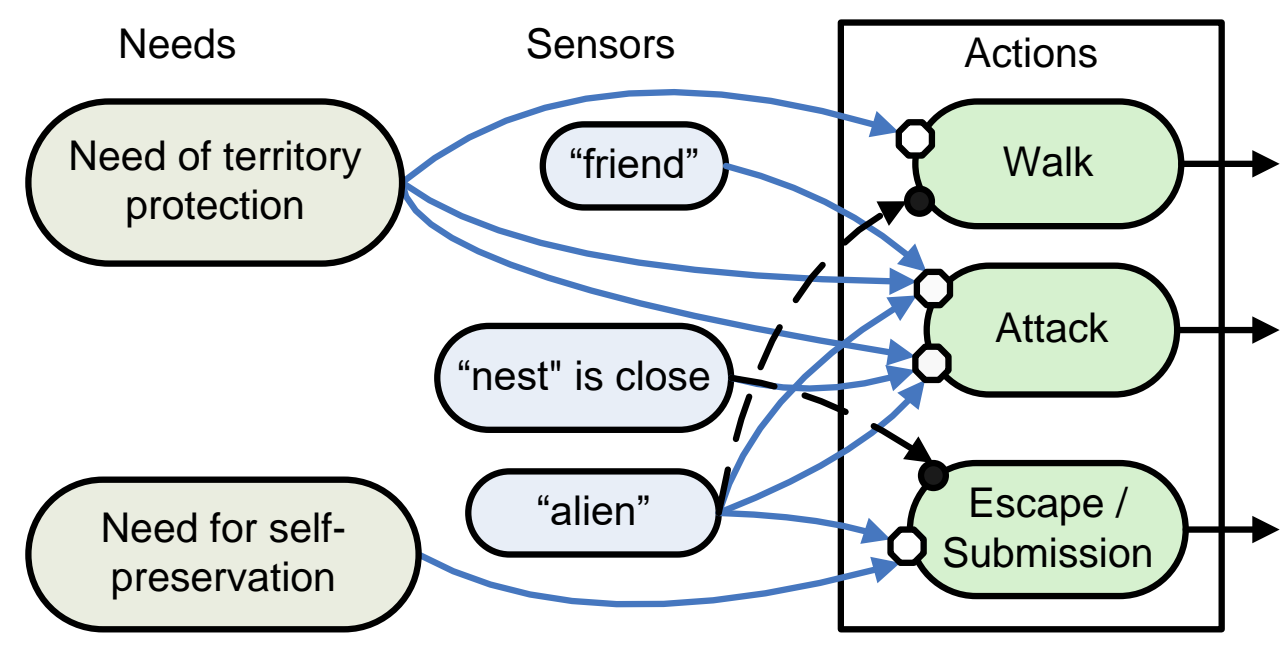

Fig. 1. Scheme of territory protection

If you do not include an aggressive element in these schemes, then agent behavior will be determined only by the magnitude of the need. For example, if the desire to dominate is greater than the need for self-preservation, then the agent will attack the opponent and runs away in other case.

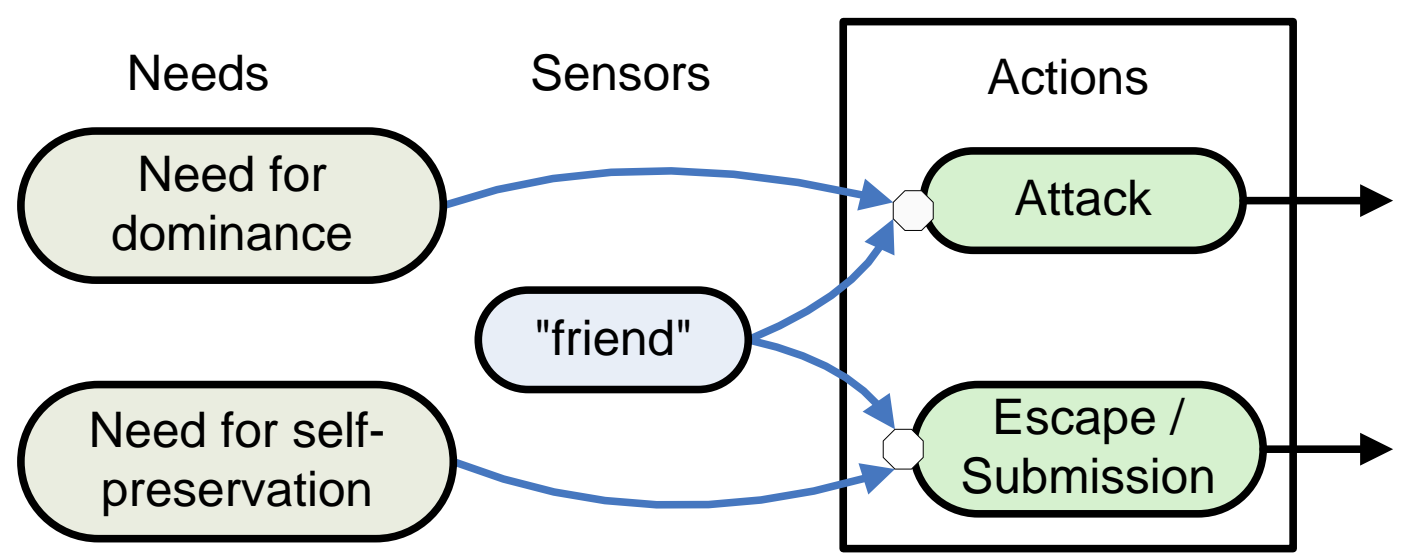

Fig. 2. Scheme of dominance

The inclusion of an aggressive component in the agent control scheme allows us to introduce a certain element of unification. This component will be responsible for assessing the current situation. The estimate can be expressed as follows. If the agent assesses the situation as a threat, he attacks the source of this threat; if he assesses it as a danger, he runs away.

A generalized scheme of behavior with an aggressive component is shown in Figure 3. Similarly, you can add additional needs to the diagram in Figure 3. An aggressive component can be technically implemented using a set of parameters. The behavior of the agent can be controlled by changing the parameter's values and not changing the control system structure. 


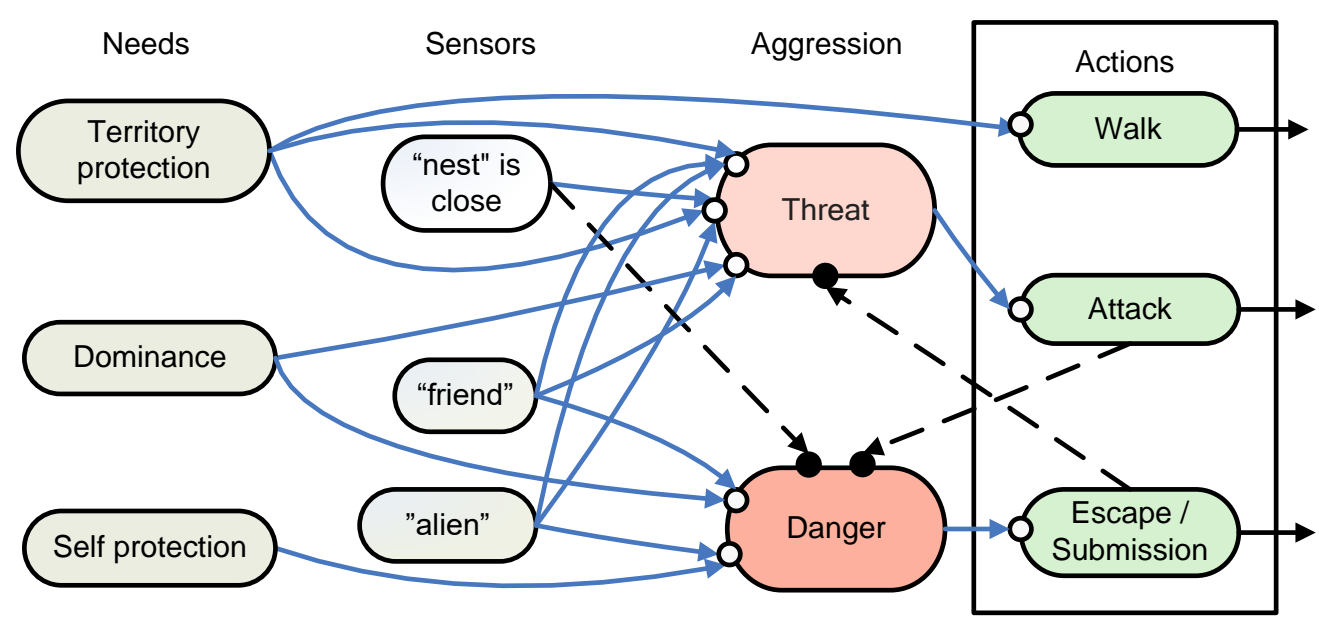

Fig. 3. General scheme of behaviors with an aggressive component

A similar approach is described in [20], where the principles of parasitic control of an agent with the emotional control system were considered. The change in the agent's behavior was achieved by changing the weights of the needs, changing the evaluation channels of the control system, and influencing the feedback loop parameters.

Neuroplasticity is the ability of the brain to change throughout an individual's life, e.g., brain activity associated with a given function can be transferred to a different location, and synapses may strengthen or weaken over time [21]. Long-term modifications of synaptic transmissions constitute the foundation of the processes by which information is stored in the central nervous system. In particular, the effect of plasticity lies in the fact that changes in the efficacy of synaptic connections can lead to a change in behavior. New conduct rules are not required in this case.

Conduct rules can be described in terms of the certainty factor $(\mathrm{CF})$ in the classical MYCIN-like production model [22]. For each rule, there is some a priori weight, which we denote by $\omega_{R}$. Then, the resulting CF of the conclusion can be defined as the product of $\omega_{R}$ on the CF of the premise $\omega_{C}$ :

$$
\omega=\omega_{R} \omega_{C}
$$

Let the values of all input signals (including sensors) be normalized on the interval $[0,1]$. Then, the "negation" can be defined as the reciprocal value $(-\omega=1-\omega)$; the "AND" and "OR" operations can be calculated as a minimum and a maximum, respectively; and with confirming rules, one can act naturally:

$$
\omega=\omega_{1}+\omega_{2}-\omega_{1}+\omega_{2}
$$

To change the behavior, it is necessary either to change the CF rules or to introduce additional fictitious sensors into the circuit, which will increase or decrease the strength of the synaptic connections. We will stop on the second option and will introduce additional sensors. They will allow us, in particular, to implement such behavior of the agent, the manifestations of which the outside observer will perceive as aggressive.

Now, we need to select the parameters that will be simulated by these fictitious sensors. The main characteristics of aggressive behavior are as follows [23]: congenital aggressiveness and the current aggressive level, which may decrease or increase depending on the animal condition, the results of its actions, etc.

Congenital aggressiveness in animals is confirmed by high indices of inheritance of aggressiveness, which are established in the study of different animal species, particularly mice [24] and birds [25]. The influence of the results of the interactions of individuals on their level of aggressiveness is confirmed by many studies. After a fight, the current aggressiveness of individuals decreases, regardless of the outcome of the conflict (the stage of appeasement is coming). However, in the next encounter with an unfamiliar partner, the animal that wins the fight is more aggressive, and the aggressiveness of the individuals suffering defeat is, contrarily, suppressed [26].

Due to the independence of these parameters, you can select them to simulate the aggressive component and implement them with a single fictive sensor A. The innate level $A_{o}$ sets the initial value of the current level $A_{c} . A_{c}$ determines the current inclination of the agent to enter the conflict. The value of $A_{c}$ increases after its victory (W $=1$ ) and decreases after defeat $(\mathrm{W}=0)$. The change in $A_{c}\left(\Delta A_{c}\right)$ is determined by the amplification factors $\alpha$ and the attenuation factors $\beta$ of the corresponding synaptic connections:

$$
\Delta A_{c}=\left\{\begin{array}{c}
1-e^{-\alpha t}, \text { if } W=1 \\
e^{-\beta t}, \text { if } W=0
\end{array}\right.
$$


$A_{c}$ will play the role of an agent's memory of the results of previous conflicts. Figure 4 shows a hybrid neuroproduction control system supplemented by a sensor-valve pair simulating a fictitious sensor A (parameter $A_{c}$ ), and two external events, namely, "victory" and "defeat." In addition to the above, the agent has needs for food and comfort and some sensors: food, obstacle, danger (light), and hunger sensors. The latter is a virtual sensor and reflects the agent's internal state. The agent is afraid of light, so he runs away from it, satisfying the need for comfort. The sensors "friend" and "alien" are combined into one "hindrance" sensor to simplify the circuit.

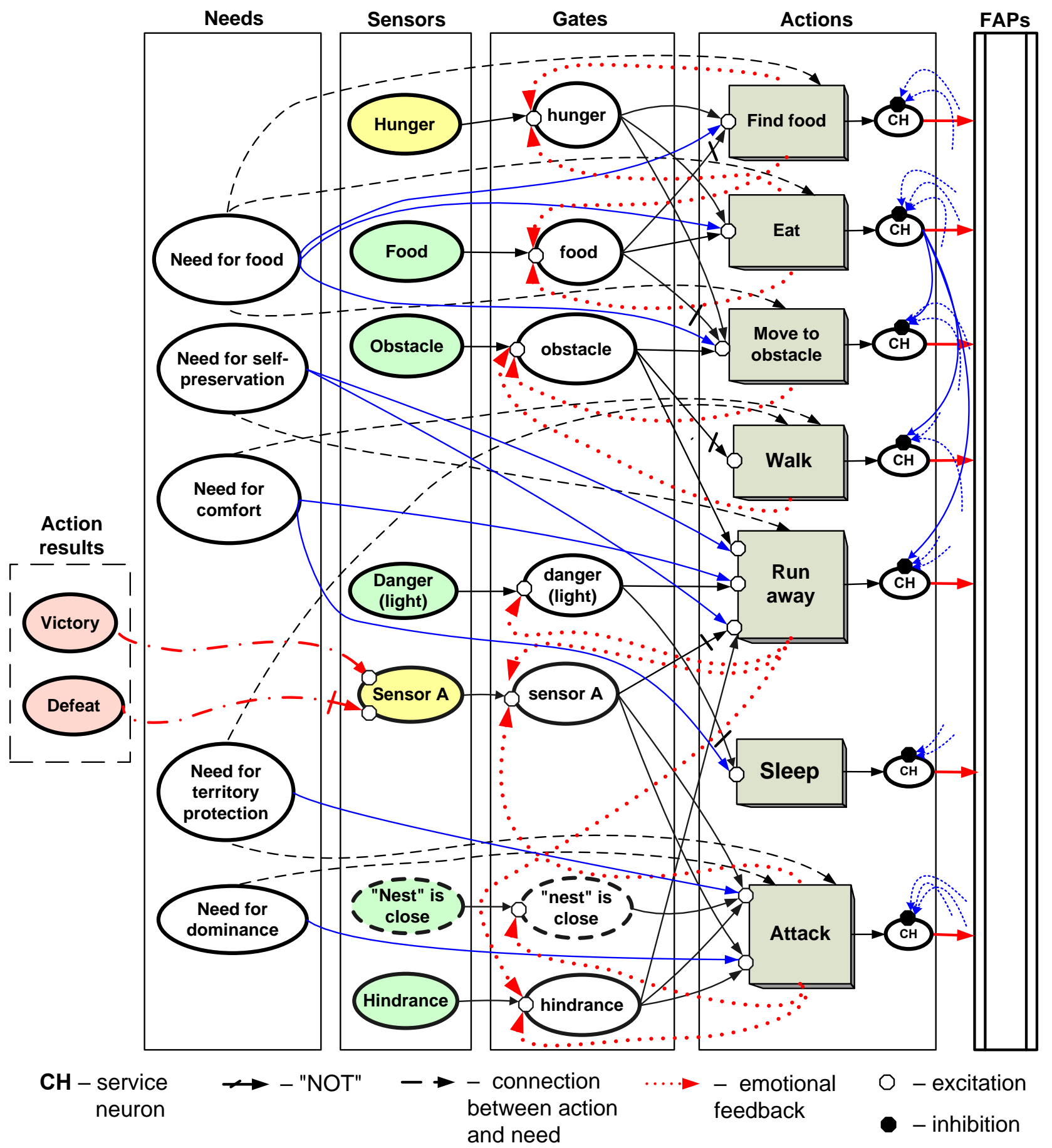

Fig. 4. The architecture of the emotional control system with an aggressive component

\section{Implementation of the general model of aggressive behavior}

Simulation modeling was carried out using the Kvorum multi-agent modeling system. In the experiments, the agent's behavior has been studied and found to reflect the needs for food, self-preservation, comfort, and domination. The agent moved around the polygon, where there were hindrances and there was food. 
When a hindrance is detected, the agent attacked it or ran away, depending on the amount of needs and its internal state $\left(A_{c}\right)$. In the attack, his win or loss was determined by the ratio of his force $F_{a}$ and the opponent's strength $F_{c}$, and it was calculated based on probability $\mathrm{p}=\mathrm{F}_{\mathrm{a}} /\left(\mathrm{F}_{\mathrm{a}}+\mathrm{F}_{\mathrm{c}}\right)$. The following problem was posed in the experiments. The change in the behavior of the agent depending on the current aggressiveness level was determined. The value of $A_{0}$, the amplification and attenuation coefficients of the synaptic connections ( $\alpha$ and $\beta$ ), and the value of the needs for selfpreservation and dominance affect $A_{c}$. The change in the agent's behavior is expressed as the ratio of the "attack" or "flight" actions.

Two series of experiments were conducted for 10 starts for each combination of the initial parameter values. Each experiment lasted 5,000 cycles of model time. The need for dominance varied from 0.2 to 0.4. To obtain more contrasting results in the first series, the opponents were significantly superior to the agent in strength. In the second series, on the contrary, he was much stronger than the opponents were. The experiment results are shown in Figure 5. Here, the coefficient $K$ is indicated on the abscissa. $K$ is the ratio of the amplification and attenuation coefficients: $\mathrm{K}=\alpha /(\alpha+\beta)$.

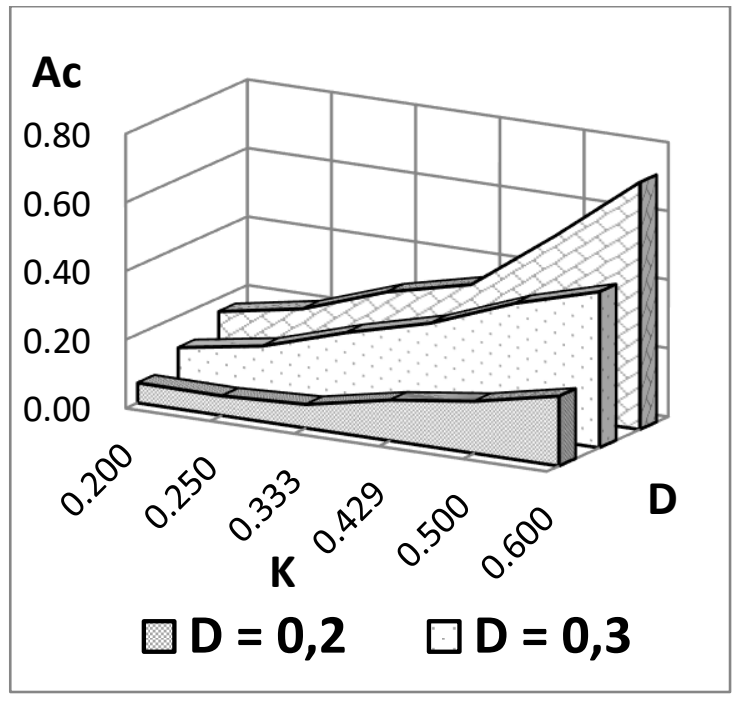

a)

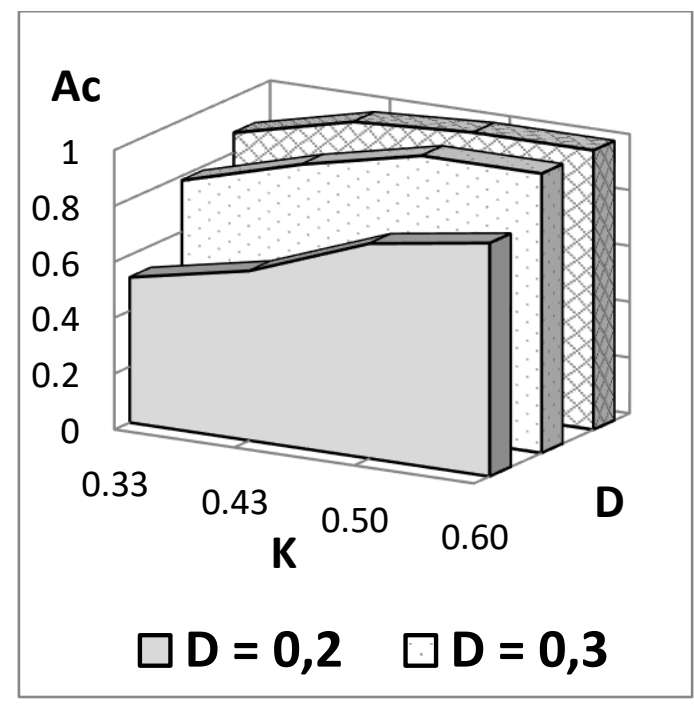

b)

Fig. 5. The results of the experiments: a) a series with weak opponents; b) a series with strong opponents

On the one hand, these experiments (Figure 5) demonstrated obvious results. An increase in the need for domination leads to an increase in the aggression level. A similar effect is exerted by an increase in the amplification coefficient $\alpha$ in comparison with the attenuation coefficient $\beta$. On the other hand, the experiments confirmed the possibility of controlling the agent's behavior with the help of the parameters introduced, and they allowed for a quantitative assessment of the investigated dependence.

The mechanism can be basis for solution of the territorial distribution problem in antagonistic environments or in environments with conflicts of interests. Mostly, such tasks are solved using some special mechanisms. For example, in [27] proposes a flocking control algorithm for a team of autonomous underwater vehicles (AUVs) deployed for group motion in a network where only local communication exists among the participating AUVs. The control scheme is based on agreement or consensus protocol. An approach based on behavior management will solve the territorial distribution problem without the use of specific protocols and additional behavior rules.

\section{Conclusion}

The proposed model of aggressive behavior accounts for previous conflict experience. It allows one to simulate the phenomenon of increasing aggressiveness with the age of the agent and the effect of forgetting one's own experience. We can control this behavior by changing the parameter values and creating more or less aggressive agents. Therefore, the additional parameter "aggressiveness" allows for the provision of a greater variety of agent behaviors depending on the state of the environment and the management of an agent or agent group, without adding superfluous behavior rules.

This mechanism is rater universal, and it can be used as a base for implementing various models of social behavior in group robotics. Its main limitations are related to the formulation of the problem to be solved in terms of behavioral reactions. In the future, the plan is to investigate group agent behavior with an aggressive component. 


\section{Acknowledgments}

The project was supported by RSF 16-11-00018 grant (control system architecture), and RFBR 17-29-07083-ofi-m grant (regulatory mechanisms for behavior management).

\section{References}

[1] Karpov, V.E. (2016) Models of social behaviour in the group robotics. Large-Scale Systems Control (Upravlenie Bol'šimi Sistemami), No 59. pp. 165-232.

[2] Tinbergen, N. (1953) Social Behavior of Animals. Methuen. 150 p.

[3] Olivier, B. \& Young, L.J. (2002) Animal models of aggression. Neuropsychopharmacology: The Fifth Generation of Progress. pp. 1699-1708. doi:10.1097/00002826-198406001-00372

[4] Brown, S., Zuluaga, M., Zhang, Y. \& Vaughan, R. (2005) Rational aggressive behaviour reduces interference in a mobile robot team. 2005 Int. Conf. Adv. Robot. ICAR '05, Proc. pp. 741-748. doi:10.1109/ICAR.2005.1507491

[5] Zhang, Y. \& Vaughan, R. (2006) Ganging up: Team-based aggression expands the population/performance envelope in a multi-robot system. Proc. - IEEE Int. Conf. Robot. Autom. pp. 589-594. doi:10.1109/ROBOT.2006.1641774

[6] Kulinich, A.A. (2016) A model of agents (robots) command behavior: The cognitive approach. Autom. Remote Control 77, pp. 510-522.

[7] Scheutz, M. \& Schermerhorn, P. (2004) The More Radical, the Better: Investigating the Utility of Aggression in the Competition among Different Agent Kinds. From Anim. to Animat. 8. Proc. 8th Int. Conf. Simul. Adapt. Behav. pp. 445-454.

[8] Karpova, I. (2018) About Realization of Aggressive Behavior Model in Group Robotics. BICA 2017. Advances in Intelligent Systems and Computing (eds. Samsonovich, A. V \& Klimov, V. V) pp.78-84 (Springer International Publishing). doi:10.1007/978-3-319-63940-6_11

[9] Lorenz, K. (2002) On Aggression. London: Routledge, 2002. 306 p.

[10] Shilov, I.A. (2002) Population homeostasis. Zool. Zhurnal 81, pp.1029-1047.

[11] Nelson, R.J. (2005) Biology of Aggression. Oxford University Press. 528 p.

[12] Butovskaya, M.L. (2002) Aggression and reconciliation as the basic properties of social systems: man and other primates (Agressiya i primirenie kak bazovye svojstva sotsial'nykh sistem: chelovek i drugie primaty). in Anthropology on the threshold of the III millennium, May 29-31, 2002. pp. 125-160.

[13] Manning, A. \& Dawkins, M.S. (1998) An Introduction to Animal Behaviour. Cambridge University Press. 450 p.

[14] Zakharov, A. A. (1991) Organization of communities in ants (Organizaciya soobshchestv u murav'ev). Nauka, 1991. $277 \mathrm{p}$.

[15] Zakharov, A.A. (1978) Muravey, sem'ya, koloniya. (Ant, family, colony). Nauka, 1978. 144 p.

[16] Caldara, M., McBride, M.T., McCarter, M.W. \& Sheremeta, R.M. (2017) A Study of the Triggers of Conflict and Emotional Reactions. Games, No 8, pp. 1-12.

[17] Karpov, V.E. (2010) Robot's Emotions (Emocii robotov) // XII Russian Conference on Artificial Intelligence KII2010 (September 20-24, 2010, Tver'): Fizmatlit, Conf. Proc., vol.3, pp. 354-368.

[18] Simonov, V.P. (1991) Thwarted action and need - informational theories of emotions. Int. J. Comp. Psychol. Vol.5. No 2. Pp.103-107.

[19] Tsetlin, M.L. (1973) Automation theory and modeling of biological systems. Volume 102 (Mathematics in Science and Engineering). Academic Press, 1973. 288 p.

[20] Karpov, V. (2017) The parasitic manipulation of an animat's behavior. Biol. Inspired Cogn. Archit. 21, pp. 67-74.

[21] Bennett, E.L., Diamond, M.C., Krech, D. (1964) Chemical and Anatomical Plasticity of the Brain. Science (80). 146, pp. 610-619.

[22] Karpov, V. (2014) Robot's temperament. Biol. Inspired Cogn. Archit. 7, pp. 76-86.

[23] Kudryavtseva, N.N., Markel, A.L. \& Orlov, Y.L. (2015) Aggressive behavior: genetic and physiological mechanisms. Russ. J. Genet. Appl. Res. 5, pp. 413-429.

[24] van Oortmerssen, G. A. \& Bakker, T. C. M. (1981) Artificial selection for short and long attack latencies in wild Mus musculus domesticus. Behav. Genet. 11, pp. 115-126.

[25] Drent, P. J., Van Oers, K. \& Van Noordwijk, A. J. (2003) Realized heritability of personalities in the great tit (Parus major). Proc. R. Soc. B Biol. Sci. 270, pp. 45-51.

[26] Hsu, Y., Earley, R. L. \& Wolf, L. L. (2006) Modulation of aggressive behaviour by fighting experience: Mechanisms and contest outcomes. Biol. Rev. Camb. Philos. Soc. 81, pp. 33-74.

[27] Subudhi B., Rayguru M.M., Filaretov V., Zuev A. Design of a consensus based flocking control of multiple autonomous underwater vehicles using sliding mode approach. Annals of DAAAM and Proceedings of the International DAAAM Symposium. 2017, pp. 4-13. DOI: 10.2507/28th.daaam.proceedings.001 